\title{
EFFECT OF SURFACE ROUGHNESS Ti6AI4V MODIFIED BY HYDROXYAPATITE COATING
}

\author{
Mohammed Alqasim ALSABTI ${ }^{1}$, Ion CIUCA $^{2}$, Bogdan ŞTEFAN VASILE ${ }^{3}$, \\ Roxana TRUSCA ${ }^{4}$, Alaa ABOU HARB ${ }^{5}$ \\ ${ }^{1} \mathrm{PhD}$ Student, Doctoral School Faculty Material Science and Engineering, \\ Polytechnic University of Bucharest, Romania, \\ ${ }^{2}$ Prof. univ. dr. ing. Faculty Material Science and Engineering, Polytechnic University of Bucharest, Romania \\ ${ }^{3}$ Ph.D. Faculty of Applied Chemistry and Materials Science, Polytechnic University of Bucharest, Romania. \\ ${ }^{4} \mathrm{PhD}$ Student, Doctoral School Engineering and Management of Technological Systems, Industrial Engineering \\ Department, Polytechnic University of Bucharest, Romania, \\ ${ }^{5} \mathrm{PhD}$ Student, Doctoral School Faculty Material Science and Engineering, \\ Polytechnic University of Bucharest, Romania, \\ e-mail: mohammedalkasim_faik@yahoo.com; ion.ciuca@medu.edu.ro; bogdan.vasile@upb.ro; \\ roxana_gheta@yahoo.com
}

\begin{abstract}
Several studies have described the use of HAp layers on titanium alloys in the recent years. Among the methods of strengthening the adherence of such layers, which call the researchers' attention, there is the development of coating by sol-gel type techniques. Coatings of HAp on the alloy of Ti6Al4V allow the formation of uniform layers, with controlled porosity and better adhesion and with properties required for its use as orthopaedic implants.

In this paper, samples of Ti6Al4V alloy were used as substrates with different surface roughness, coated with HAp layers that were deposited by a spin-coating technique, using a solution obtained by the sol-gel method. The layers have good homogeneity and good adhesion to the substrate, where stability and uniformity observes for Hap_250 and Hap_110 compared with Hap_29 and Hap_45, that indicate to the importance of surface roughness. Therefore, the biological characterization was investigated, including the film stability in the Simulated Body Fluid (SBF) and the antimicrobial activity of Ti6Al4V alloys coated with hydroxyapatite. Also, the biofilm formation by P. aeruginosa species was evaluated. The morphology and structure of the films were performed by scanning electron microscopy (SEM) and X-ray diffraction $(X R D)$.
\end{abstract}

KEYWORDS: Hydroxyapatite, thin film, Ti6Al4V alloy, XRD, SEM

\section{Introduction}

The mineral phase of the bone similar to hydroxyapatite HA synthetic, of chemical formula $\left[\mathrm{Ca}_{10}\left(\mathrm{PO}_{4}\right)_{6}(\mathrm{OH})_{2}\right]$, has been widely studied and used in the field of medicine as a biomaterial because its excellent biocompatibility characteristics promote its acceptance and adequate osseointegration in the biological environment [1].

Calcium phosphates are the most important mineral phase in the hard tissues of vertebrates, and since the beginning of the $20^{\text {th }}$ century, different studies have demonstrated the similarity between bone minerals and calcium phosphate minerals with an apatite structure.

As a consequence, these calcium phosphates of natural or synthetic origin are one of the most used routes in bone or dental surgery today, when a supply of material is necessary [2].

There are, according to their physical chemistry, three families of calcium phosphates (metaphosphates, pyrophosphates, and orthophosphates), and within this last family, different subfamilies, according to their $\mathrm{Ca} / \mathrm{P}$ ratio (TTCP-tetracalcium phosphate-, Hap hydroxyapatite-, TCP)- tricalcium phosphates-, OCPoctacalcium phosphate-, etc.), but of all of them, the 
material used in prosthesis coatings, par excellence, is hydroxyapatite [3].

Apatites are a family of ionic compounds described by the chemical formula $\mathrm{M}_{10}\left(\mathrm{RO}_{4}\right)_{6} \mathrm{X}_{2}$ (where $\mathrm{M}=\mathrm{Ca} 2+, \mathrm{Pb} 2+, \mathrm{Na}+$, etc., $\mathrm{X}=\mathrm{OH}-, \mathrm{F}-, \mathrm{Cl}=$, etc.; $\mathrm{RO} 4$ = PO43- , AsO43-,VO43-, etc.). The X will be the one that gives the name to the apatite. In hard tissues, the apatite present is hydroxyapatite; that is, an apatite where the $\mathrm{X}$ is an $\mathrm{OH}-$. Thus, the formula of the stoichiometric hydroxyapatite (calcium), with a hexagonal crystalline structure, would be $\mathrm{Ca}_{10}\left(\mathrm{PO}_{4}\right)_{6}(\mathrm{OH})_{2}[4,5]$.

The recent years have witnessed the use of HAp layers on titanium alloys. Among the methods of strengthening the adherence of such layers, which draw the attention of researchers, there is the development of coating by sol-gel type techniques. Coatings of HAp on the alloy of Ti6Al4V allow the formation of uniform layers with controlled porosity, better adhesion and protective effect to the phenomenon of corrosion, having properties that recommend its use as orthopaedic implants [6].

\section{Materials and methods}

\subsection{Selection of Materials and the Coating Process}

This section of the article covers the materials selected for synthesis and deposition. Titanium alloy (Ti6Al4V) is in the form of plates of about $5 \times 5 \mathrm{~mm}$ with a thickness of 2 and $0.5 \mathrm{~mm}$ respectively. These plates were further processed by using particles of $\mathrm{Al}_{2} \mathrm{O}_{3}$ blasting process with differently sized particles (29, 45, 110 and $250 \mu \mathrm{m}$ respectively) to produce different surface roughness for each sample.

The preparation of hydroxyapatite is made as follows: a high concentration of Triethylphosphate 98\% $\mathrm{P}\left(\mathrm{OCH}_{2} \mathrm{CH}_{3}\right)_{3}$ (Sigma-Aldrich Inc., St. Louis, $\mathrm{MO})$, Ethanol $\left(\mathrm{CH}_{3} \mathrm{CH}_{2} \mathrm{OH}\right)$, deionized water and Calcium nitrate hydrate $99 \%$ $\left(\mathrm{Ca}\left(\mathrm{NO}_{3}\right)_{2} \cdot 4 \mathrm{H}_{2} \mathrm{O}\right)($ Sigma-Aldrich Inc., St. Louis, $\mathrm{MO}$ ), and Ammonia solution were used to obtain the solution for sol-gel method, coupled with the spin coating technique. The equipment used to obtain the coated samples is A Spin Coater from Laurell model WS-650.

The obtained samples of Ti6Al4V, coated with hydroxyapatite samples investigated via SEM (coupled with EDX), XRD. In addition, stability tests were made in SBF. All samples were subjected to microbiological characterization and cell culture testing for biocompatibility issues.

Figure 1 shows the technological flow of synthesis of Ti6Al4V coated alloys with hydroxyapatite (HAp) and their characterization.

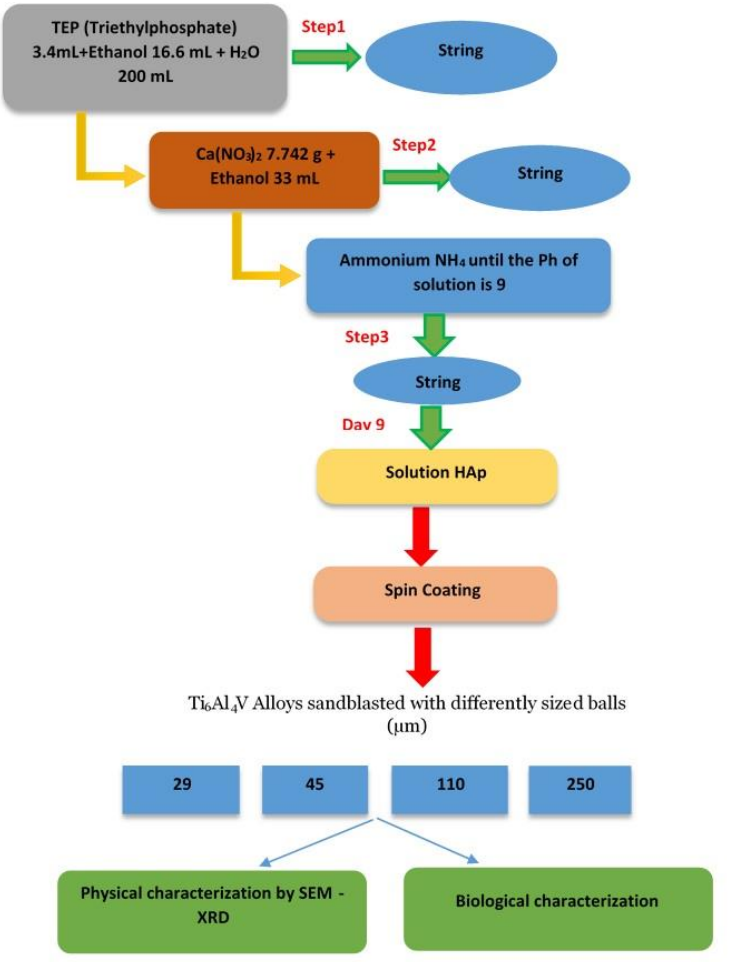

Fig. 1. shows the technological workflow

\subsection{Preparation of materials to coated process}

For the first step in the synthesis of HAp, the solution was prepared as follows: i) distilled water was added to $1 \mathrm{M}$ solution of Triethylphosphate (TEP) in ethanol and allowed for 24 hours to be homogenized under continuous stirring; ii) after 24 hours, the TEP solution was added to $1 \mathrm{M}$ of calcium nitrate solution in ethanol, maintaining a $\mathrm{Ca} / \mathrm{P}$ ratio of 1.67, and homogenized for 24 hours. In this step, the $\mathrm{pH}$ was maintained at a value of 9 by ammonia solution addition and finally, iii) the obtained solution was left to homogenize for 7 days.

After the solution was obtained, Ti6Al4V alloys were coated using a spin coater machine. The samples were coated with 20 thin layers of Hap solution at a speed of $2000 \mathrm{rpm}$ for 5 seconds. After every deposited layer, the films were dried at $80{ }^{\circ} \mathrm{C}$ for 10 min on a heating plate. The sample code obtained is shown in Table 1 below:

Table 1. Code of Ti6Al4V coated with HAp

\begin{tabular}{|c|c|c|}
\hline Code & Alloy & Blasting particles size $(\boldsymbol{\mu m})$ \\
\hline HAp_29 & Ti6Al4V & 29 \\
\hline HAp_45 & Ti6A14V & 45 \\
\hline HAp_110 & Ti6Al4V & 110 \\
\hline HAp_250 & Ti6A14V & 250 \\
\hline
\end{tabular}


The morphology and microstructure of the films were observed with an electronic scanning microscope (SEM) Quanta Inspect F, which had been purchased from the FEI, the resulting images being made by recording the resultant secondary electron beam with $30 \mathrm{keV}$ energy. The crystal structure of the coated films was identified using a PANalytical Empyrean model diffractometer equipped with a hybrid monochromator (2xGe 220) on the side and a parallel plate collimator mounted on the PIXcel 3D detector on the diffracted side. Grazing Incidence Xray Diffraction (GIXRD) measurements were performed at room temperature with an incidence angle of $\omega=0.5^{\circ}$ for Bragg angle values of 20 between $10^{\circ}$ and $80^{\circ}$, using $\mathrm{Cu} \mathrm{K \alpha}$ radiation with $\lambda=$ $1.5406 \AA$ (40 mA and $45 \mathrm{kV})$. The elemental composition of the coating was identified using Energy Dispersive Spectroscopy (EDS).

\section{Results and Discussion}

\subsection{X-ray characterization (XRD)}

In Figure 2, the X-ray diffraction spectra of the uncoated Titanium alloy (Ti6Al4V) can be observed. By analysing the spectra for the uncoated sample, we can see that it has the peaks which are all attributed to $\alpha$ phase titanium, and there is no $\beta$ phase diffraction peak in the alloy.

Thus, the X-ray diffraction spectra of the coated Titanium alloy (Ti6Al4V) can be observed. The Xray diffraction spectra of Hap_29 and Hap_45, with roughness obtained by using particles of $29 \mu \mathrm{m}$ and $45 \mu \mathrm{m}$ respectively, have a phosphatic ceramic film with a rhombohedral structure, composed of $\beta$ tricalcium phosphate $\mathrm{Ca}_{3}\left(\mathrm{PO}_{4}\right)_{2}$ in the hexagonal form, according to ASTM sheets 04-010-0295.

It should be noted that the samples are well crystallized in both cases but also that the intensity of diffraction lines for $\mathrm{Ca}_{3}\left(\mathrm{PO}_{4}\right)_{2}$ decreases with increased roughness, which is induced by the particle with $45 \mu \mathrm{m}$. This can be attributed to the fact that Tricalcium diphosphate (TCP) $\mathrm{Ca}_{3}\left(\mathrm{PO}_{4}\right)_{2}$ was formed in holes due to the larger size of the particles.

In the case of diffraction spectra obtained for HAp_110 and HAp_250, one can see that the samples exhibit lower crystallinity compared to HAp_29 and HAp_45, which is demonstrated by the intensity of smaller high diffraction lines. Both XRD Spectra reveal that the layer obtained and analysed for the surface of titanium alloys is $\mathrm{Ca}_{5}\left(\mathrm{PO}_{4}\right)_{3}(\mathrm{OH})$ and is formed according to ASTM datasheets 00-009-0432 with Hexagonal structure, indicating that deposition has been successful.

In all samples Ti is identified from the substrate ASTM 04-010-0295. In addition, Figure 2 a, b, c, d, and e shows a compression between Ti6Al4V alloy uncoated and Ti6Al4V alloy coated with HAp, where different structures of $\mathrm{Ca}_{5}\left(\mathrm{PO}_{4}\right)_{3}(\mathrm{OH})$ (HAp) and $\mathrm{Ca}_{3}\left(\mathrm{PO}_{4}\right)_{2}$ (TCP) are formed after coating as a result of the roughness obtained by using particles $[7,8]$.
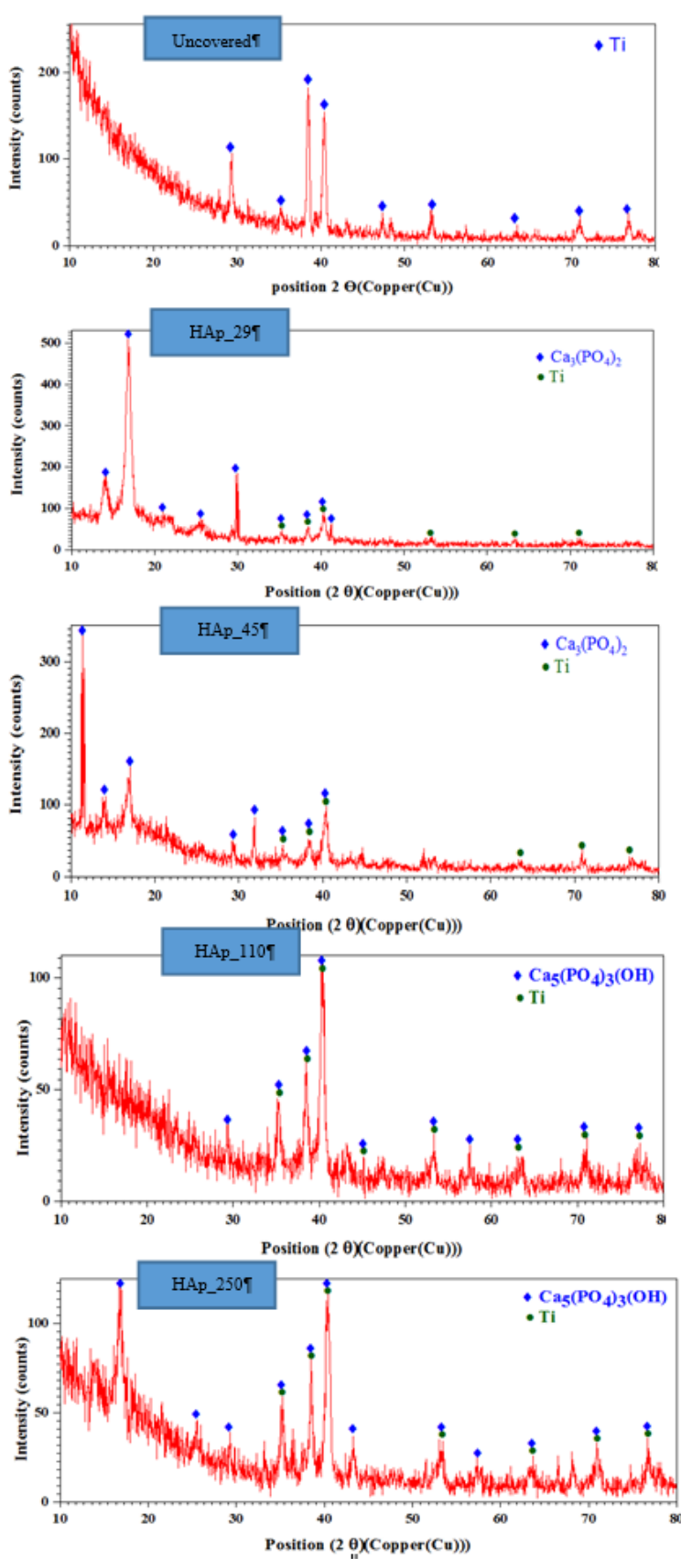

Fig. 2. shows the compared XRD Spectra for all samples of Ti6Al4V uncovered and covered HAp with different roughness

The importance of the HAp stability (HAp_250 and HAp_100) layer is higher than that of the TCP (HAp_45 and HAp_29) layer, although the TCP is more soluble than the $\mathrm{HA}$ in physiological $\mathrm{pH}$ and 
therefore more susceptible to biological radiation. But high solubility leads to early failure of TCP coating, due to high solubility, which is unpredictable. Moreover, HA has a strong inhibition of growth, better than TCP.

\subsection{Electronic Scanning (SEM)}

Electronic Scanning Microscopy (SEM) is an investigative method that can provide relevant information about the structure and state of the material surfaces. This analysis was used to
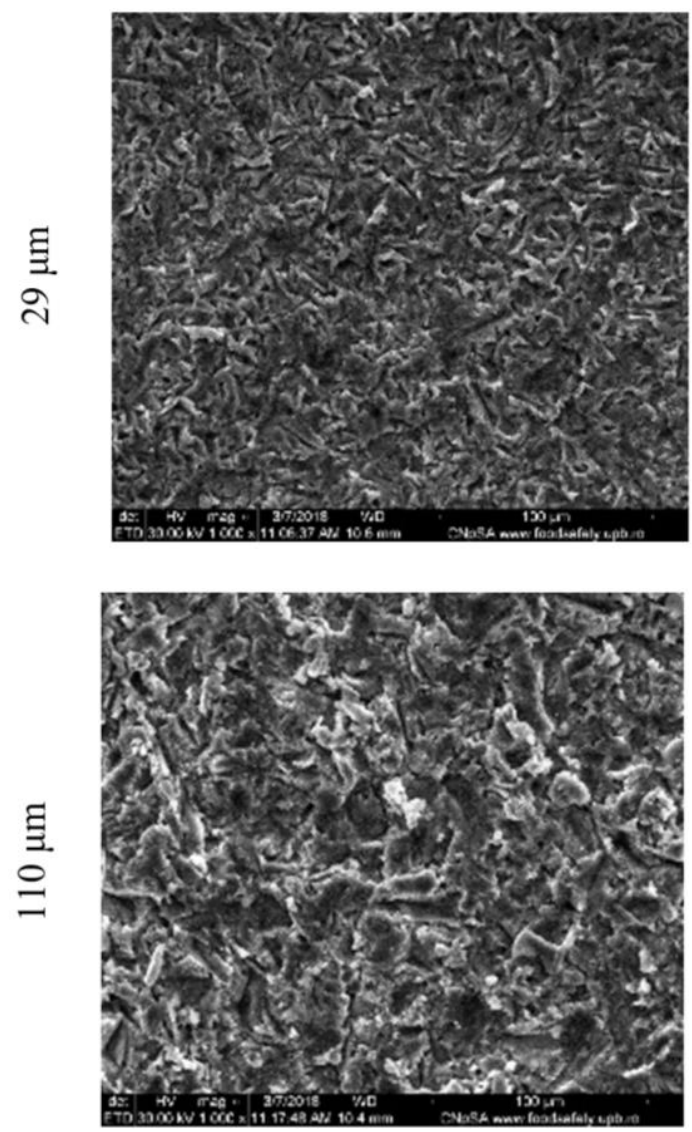

investigate the morphological characteristics of the hydroxyapatite, hydroxyapatite with silver, respectively the film deposited Ti6Al4V surfaces

\subsubsection{SEM of uncoated Ti6Al4V}

Increased surface roughness was observed as the size of particles increased through the surface morphology of titanium samples, with different surface roughness as shown in Figure 3, which presents the SEM data obtained.
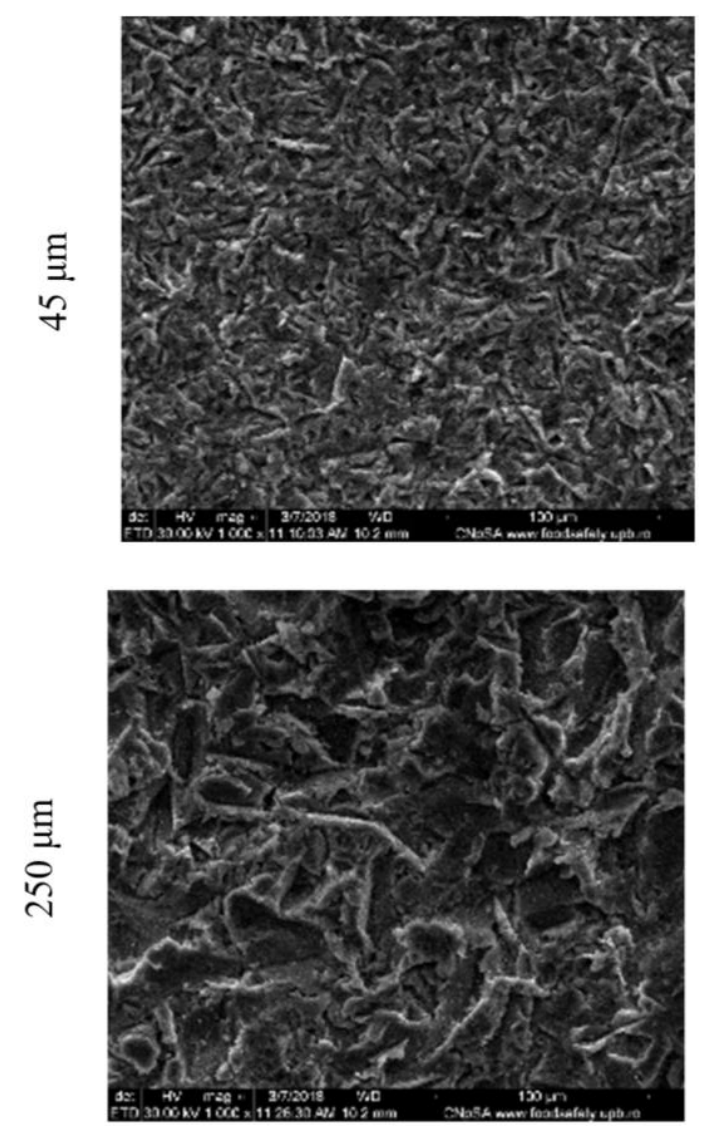

Fig. 3. shows SEM micrographs of Ti6Al4V with different roughness

\subsubsection{SEM analysis of undoped samples} HAp_29, HAp_45, HAp_110, HAp_250

The micrographs obtained of the samples HAp_29, HAp_45, HAp_110, HAp_250 show that the deposited layer on the surface of titanium support is uniform and distributed in all the recesses formed by particle halls of different sizes $(29,45,110,250$ $\mu \mathrm{m})$.

The HAp_110 and HAp_250 samples have a deposition of HAP layer morphology present in polyhedral forms because in this case HAp is formed not of $\mathrm{Ca}\left(\mathrm{PO}_{4}\right)_{2} \mathrm{OH}$.
The EDXS analysis, performed on the titanium alloy samples with HAp layers, which have been deposited using the spin coating method simultaneously, confirms the uniform distribution of the specific species found, such as calcium, phosphorus, and oxygen, characteristic of the solution submitted, as illustrated in Figure 4. 


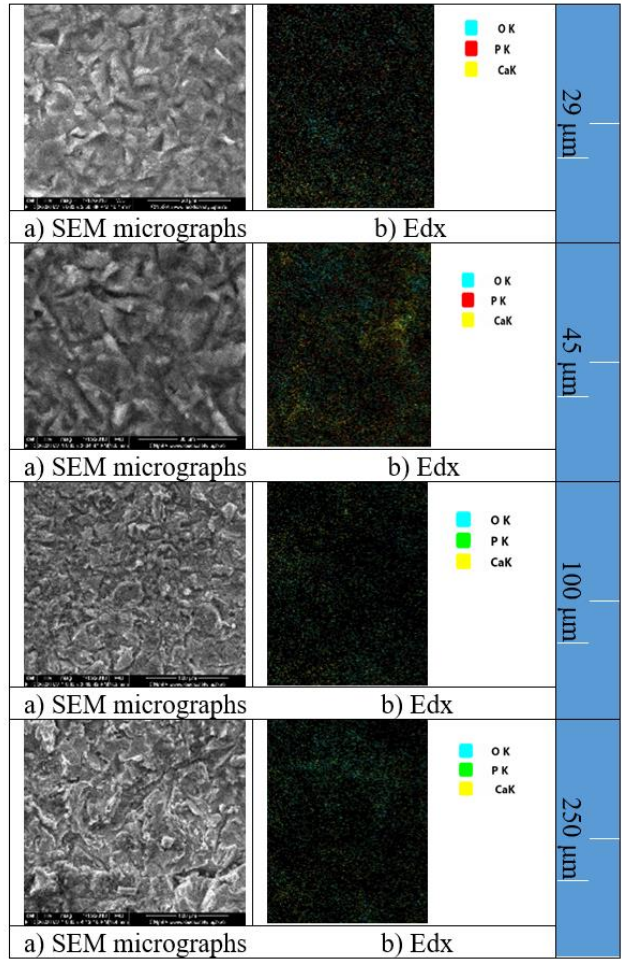

Fig. 4. shows the SEM micrograph and EDX for HAp with different roughness

\subsection{Stabilization of thin films in SBF}

The method of coating with HAp can be based on practical bio-mimicry by immersing implants in the simulated body fluid (SBF), which is an equal inorganic composition with the $\mathrm{pH}$ and temperature of the human blood plasma.

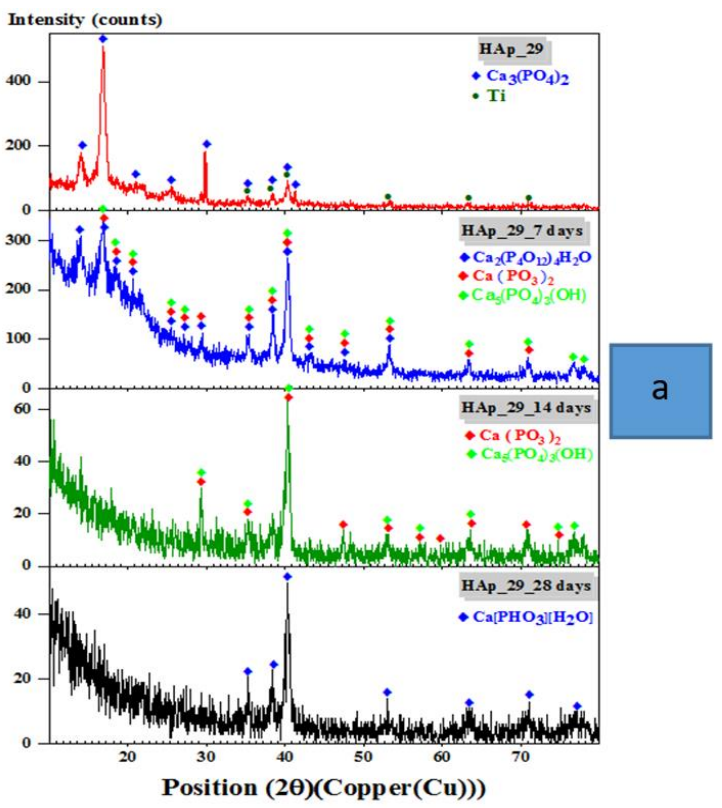

The surface morphology of the spinning coated specimens was observed by a field scanning electron microscope and X-ray spectra (XRD) analysis was performed.

\subsubsection{X-ray Diffraction Analysis of samples} HAp_29, HAp_45, HAp_110, HAp_250 after 7, 14, 28 days of immersion in SBF

In Figure 5, the X-ray diffraction spectra of HAp 29 and HAp 45, with roughness obtained by using particles of $29 \mu \mathrm{m}$ and $45 \mu \mathrm{m}$ respectively, have had a phosphorus ceramic film with Monoclinic crystal of Calcium Phosphate Hydrate $\left(\mathrm{Ca}_{2}\left(\mathrm{P}_{4} \mathrm{O}_{12}\right) \cdot 4 \mathrm{H}_{2} \mathrm{O}\right.$ and Calcium Phosphate $\left(\mathrm{Ca}\left(\mathrm{PO}_{3}\right)_{2}\right)$.

With HAp_29, it crystallized with Calcium Phosphate Hydroxide $\mathrm{Ca}_{5}\left(\mathrm{PO}_{4}\right)_{3}(\mathrm{OH})$, While the intensity of diffraction lines for $\left(\mathrm{Ca}_{2}\left(\mathrm{P}_{4} \mathrm{O}_{12}\right) \cdot 4 \mathrm{H}_{2} \mathrm{O}\right.$ of HAp_45 indicates to the decrease of crystallization compared with Hap_29.

In the case of HAp_110 and HAp_250, it can be seen that the samples exhibit lower crystallinity compared to HAp_29 and HAp_45, which is demonstrated by the intensity of smaller high diffraction lines.

The hexagonal structure of Apatite $\mathrm{Ca}_{5}\left(\mathrm{PO}_{4}\right)_{3}(\mathrm{OH})$ afford the stability of hydroxyapatite as an excellent biomaterial used for bone repair or implants.

With immersion in SBF solution, HAp_29, HAp_45, HAp_110, and HAp_250 were converted into a more stable HAp. By increasing of immersion time to 28 days, HAp peaks have risen, which confirms the growth of HAp.

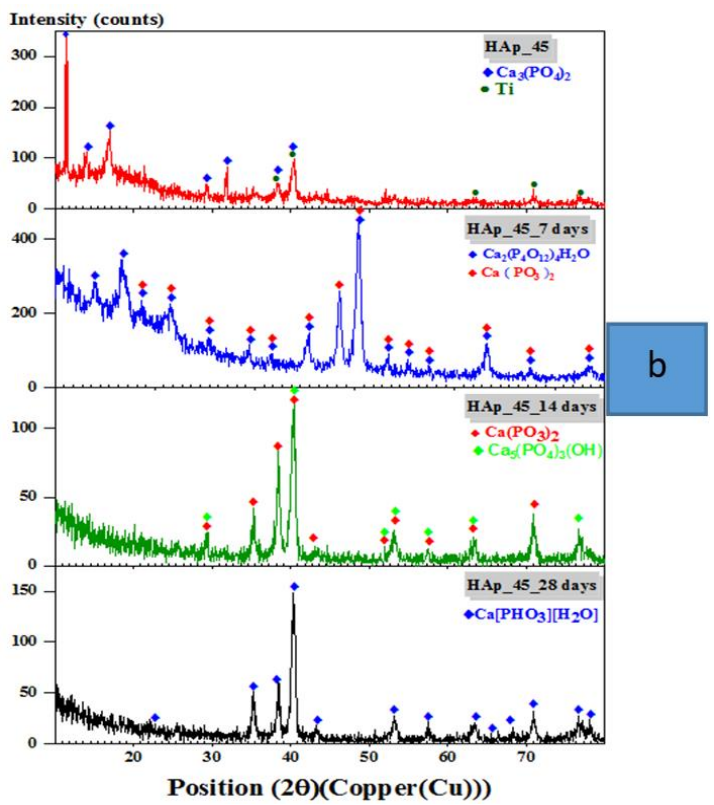



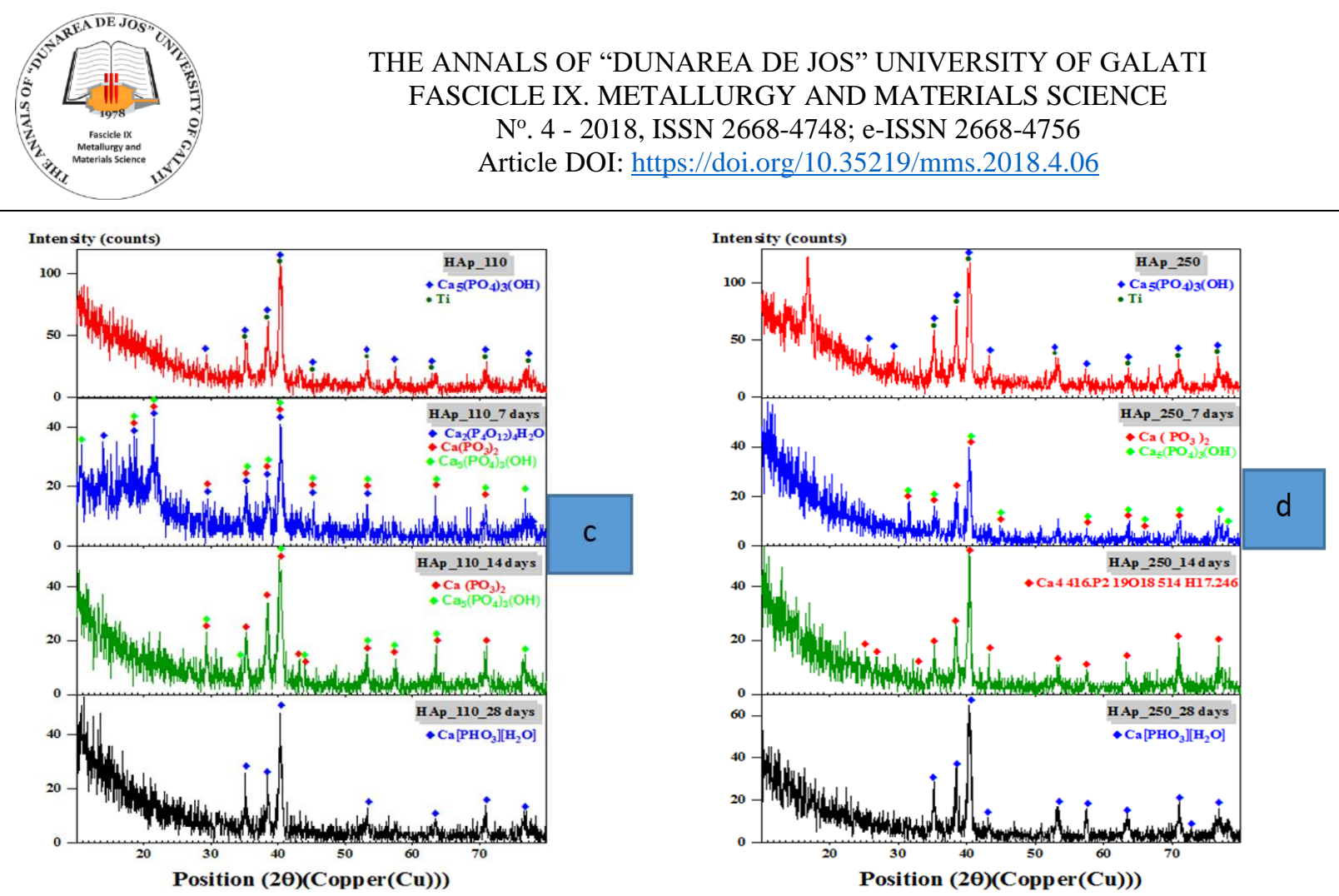

Fig. 5. shows the X-ray Diffraction, registered for samples a) HAp_29, b) HAp_45, c) HAp_110,d) HAp_250, after immersion in SBF for 0, 7, 14 and 28 days respectively

\subsubsection{Scanning Electron Microscopy (SEM)}

In Figure 6, according to SEM micrographs which were performed at 7 days after immersion in $\mathrm{SBF}$, it can be seen that the HAp film has developed quantitatively, presenting, at the same time, a homogeneous arrangement on the surface of the titanium alloys, which is an indication of high activity of the hydroxyapatite in contact with the physiological fluid in the human body.
According to the SEM micrographs obtained after 14 days of immersion in SBF, the HAp film has a more significant development on the surface of the titanium alloy substrate than the micrographs obtained at 7 days after immersion.

Via the analysis of samples after 28 days immersion in SBF, emphasized the success of hydroxyapatite deposition as homogeneous and agglomeration.
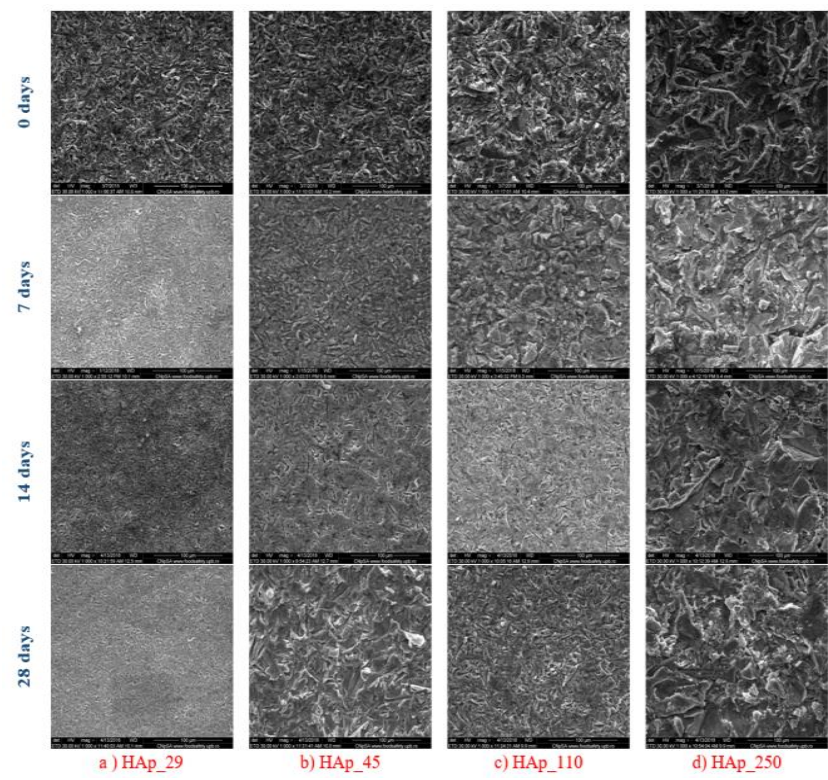

Fig. 6. shows the SEM micrographs, registered for samples HAp_29a), HAp_45 b), HAp_110 c), HAp_250 d) after 0, 7, 14 and 28 days respectively 


\subsection{Biological characterization}

The effect of the materials obtained on the growth of microorganisms in liquid media (planktonic cultures), and the effect of the surfaces obtained on the production of biofilms were tested in order to establish the antimicrobial activity of titanium alloys coated with hydroxyapatite. the numbering of the sample CODs, shown on the images taken during the biological analysis, as shown in Figure 7:

HAP 29, 6. HAP 45, 7. HAP 110, 8. HAP 250, 9. CONTROL TI, 10. P. aeruginosa ctrl.

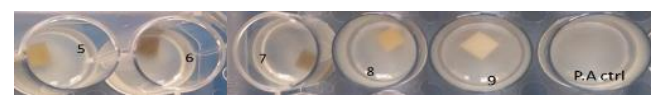

Fig. 7. shows the CODS samples used with Planktonic cultivation

\subsubsection{Growth of planktonic microorganisms in the presence of materials}

To test the effect of the materials obtained on the growth of microorganisms in liquid media (planktonic cultures), the materials obtained were sterilized by exposure to UV radiation for 20 minutes on each side. The material (sterile fragment) was individually put into a well with 6 sterile plate. Over the deposited materials, were added $2 \mathrm{~mL}$ of liquid medium (simple broth) and then $50 \mu \mathrm{L}$ of McFarland microbial suspension (Bacteria) with density $=0.5$. The 6 plates prepared were incubated at $37{ }^{\circ} \mathrm{C}$ for 24 hours. After the expired of incubation time, $200 \mu \mathrm{L}$ of the obtained microbial suspensions were transferred to 96 sterile plates and the turbidity of the microbial cultures was measured spectrophotometrically (UV) at $600 \mathrm{~nm}$.

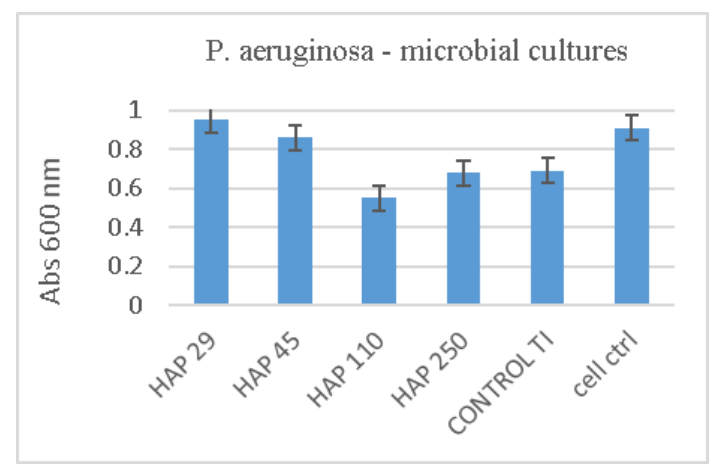

Fig. 8. shows the Abs values at $600 \mathrm{~nm}$ suggesting the growth of planktonic microorganisms in the presence of Ti-based materials
For samples 3 and 4 no microbial growth was detected visually or spectrophotometrically, which demonstrates the high antimicrobial effect of the obtained materials.

When the spectrophotometric investigation completed, it can be seen from the graph that the samples have an inhibitory effect on bacterial action on the P. aeruginosa strain as seen in Figure 8. HAp110 and HAp250. That means the roughness of the materials obtained by using particles of dimensions 110 and $250 \mu \mathrm{m}$, have positive influences on the antibacterial activity for the samples on the P. aeruginosa strain.

\subsubsection{Biofilm product}

To test the effect of the surfaces obtained on the production of biofilms, the materials obtained were sterilized by exposure to UV radiation for 20 minutes. After one by one sterile material fragment was individually deposited in a well of a 6 sterile plate. Over the deposited materials, $2 \mathrm{~mL}$ of liquid medium (plain bullion) and subsequently $50 \mu \mathrm{L}$ of McFarland (density 0.5 ) microbial suspension were added to the plates. The 6 plates still prepared were incubated at $37{ }^{\circ} \mathrm{C}$ for $24 \mathrm{~h}, 48 \mathrm{~h}$ or $72 \mathrm{~h}$. After incubation, the materials were washed with AFS (physiological saline water). After the expiration of each incubation period, the sample on which the biofilm was developed was washed with AFS and deposited in a sterile tube in $1 \mathrm{~mL}$ of AFS. The tube was vortexed vigorously for 30 seconds to detach the cells from the biofilm. The cell suspension obtained was diluted and various dilutions were seeded on solid culture media plates to obtain and quantitate the number of colonies forming units (UFC / $\mathrm{mL}$ ). Sow plates were grown for $24 \mathrm{~h}$ and the number of UFC / $\mathrm{mL}$ was calculated based on the number of viable colonies and the dilution taken into account.

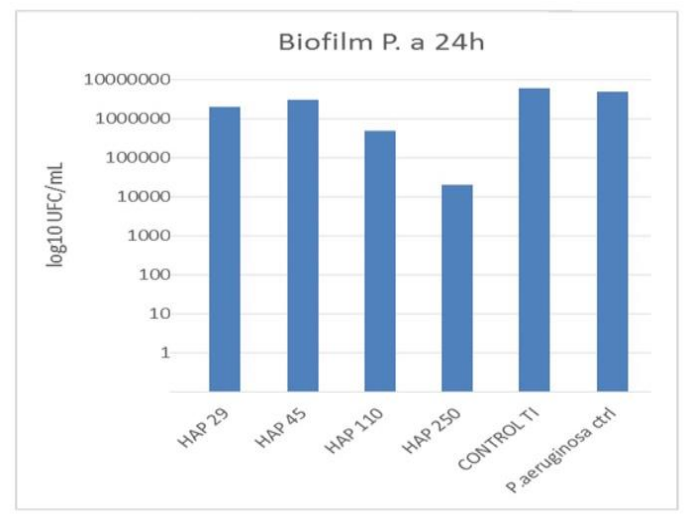

Fig. 9. Graphical representation of $U F C / m L$ values, representing the development of biofilms on the tested surfaces 
It can be seen from the Figure 9 some inhibitory effect being observed in samples 3 and 4 on the formation of monospecific biofilms by P. aeruginosa species, indicated to most significant effects of the high roughness of surface on hydroxyapatite coating which inhibitory of their development.

\section{Conclusions}

The samples HAp_29 and HAp_45 are well crystallized in both cases, however, the intensity of diffraction lines for $\mathrm{Ca}_{3}\left(\mathrm{PO}_{4}\right)_{2}$ also decreases with increased roughness, which induced by the particles with $45 \mu \mathrm{m}$. This can be attributed to the fact that Tricalcium diphosphate (TCP) $\mathrm{Ca}_{3}\left(\mathrm{PO}_{4}\right)_{2}$ was formed in holes due to the larger size of the particles.

HA_110 and HAp_250 can be seen to exhibit lower crystallinity compared to HAp_29 and HAp_45, which is demonstrated by the intensity of smaller high diffraction lines.

The hexagonal structure of Apatite $\mathrm{Ca}_{5}\left(\mathrm{PO}_{4}\right)_{3}(\mathrm{OH})$ afford the stability of hydroxyapatite as an excellent biomaterial used for bone repair or implants.

The deposited layer formed on the surface of titanium support is uniform and distributed in all the recesses.

EDX analysis, performed on titanium alloy samples, the samples with phosphate ceramic layers, which have been deposited by using the spin coating method simultaneously, confirm the uniform distribution of the specific species found, such as calcium, phosphorus, and oxygen.

The roughness of the materials obtained by using particles of dimensions 110 and $250 \mu \mathrm{m}$ have a positively influences of the antibacterial activity and biofilms activity for the samples on the P. aeruginosa strain.

\section{References}

[1]. Yilmaz B., Alshemary A. Z., Evis Z., Co-doped hydroxyapatites as potential materials for biomedical applications, Microchem. J., vol. 144, no. July 2018, p. 443-453, https://doi.org/10.1016/j.microc.2018.10.007, 2019.

[2]. Combes C., Cazalbou S., Rey C., Apatite Biominerals, Minerals, vol. 6, no. 2, p. 34, 2016.

[3]. Canillas M., Pena P., De Aza A. H., Rodríguez M. A., Calcium phosphates for biomedical applications, Bol. la Soc. Esp. Ceram. y Vidr., vol. 56, no. 3, p. 91-112, 2017.

[4]. Deng T., Xia Z., Ding H., Effect of [PO4]3-/[VO4]3-substitution on the structure and luminescence properties of Ca5[(P,V)O4)]3F:Eu3+ phosphors, Chem. Phys. Lett., vol. 637, p. 67-70, 2015.

[5]. Gallo J., Langova K., Havranek V., Cechova I., Poor survival of $A B G$ I hip prosthesis in younger patients, Biomed. Pap. Med. Fac. Univ. Palacky. Olomouc. Czech. Repub., vol. 152, no. 1, p. $163-168,2008$

[6]. Liu F., Song Y., Wang F., Shimizu T., Igarashi K., Zhao L., Formation characterization of hydroxyapatite on titanium by microarc oxidation and hydrothermal treatment, J. Biosci. Bioeng., vol. 100, no. 1, p. 100-104, 2005.

[7]. Al-Sanabani J. S., Madfa A. A., Al-Sanabani F. A., Application of calcium phosphate materials in dentistry, Int. J. Biomater., vol. 2013, no. May 2013.

[8]. Santos E. A., Farina M., Soares G. A., Anselme K., Chemical and topographical influence of hydroxyapatite and $b$ tricalcium phosphate surfaces on human osteoblastic cell behavior, 2008 . 